\title{
CHURG-STRAUSS SYNDROME WITHOUT ATOPIC COMPONENTS: THE PECULIARITY OF RAROPATHY
}

Gabriella Luiza de Jesus Costa ${ }^{1, \star}$, Karine Soares de Sousa ${ }^{1}$, Pedro Fonseca Abdo Rocha ${ }^{1}$, Andrea Carla Ribeiro da Silva ${ }^{1}$, Cicero Gonzaga Santos ${ }^{1}$, Elisa Maria Reis dos Santos ${ }^{1}$, Jessica de Cassia Vaz Oliveira ${ }^{1}$, Maria Leticia Lima Madureira ${ }^{1}$, Hanna Gonçalves de Carvalho ${ }^{1}$, Larissa Andrade Figueiredo ${ }^{1}$, Matheus Luiz Lopes Amaral ${ }^{1}$, Pedro Guido Soares Andrade ${ }^{1}$, Raiza Randazzo de Carvalho Rocha ${ }^{1}$

1.Hospital Semper, Belo Horizonte (MG), Brazil.

*Corresponding author: gabiljcosta@hotmail.com

\section{BACKGROUND}

Eosinophilic granulomatosis with polyangiitis (EGPA), which was formerly called Churg-Strauss syndrome (SCS), is classified as small and medium-sized arteries vasculitis. The most common characteristics of EGPA are asthma, nasal and sinus symptoms, and peripheral neuropathy. It is a systemic disease: skin involvement, pulmonary opacities, kidney disease, and gastrointestinal involvement may be present. It may also have constitutional and nonspecific symptoms such as weight loss, fever, and myalgia. The clinical characteristics of EGPA usually develop in several sequential phases: prodromal, eosinophilic, vasculitic. Its etiology is undetermined, but there seems to be an important allergic and immunomediated component. Diagnosis is difficult in some cases, not only because of the rarity, but also because of the clinical and pathological overlap of the different vasculitis. All these factors may result in an underestimated prevalence.

\section{CASE REPORT}

S.R.S., male, 62 years old. He presented pain and edema in lower limbs, paresthesia of hands and feet associated with hyporexia, weight loss and subfebrile state. He referred history of sinusitis of repetition and nasal polyposis, with use of several courses of antibiotics including venous. Tomography of the sinuses of the face evidencing pansinusitis, obstruction of all drainage ways of the paranasal sinuses and sinonasal polyposis. He denied history of asthma or other atopies. Blood count: leukocytosis 20,190 with severe eosinophilia 5,855. Inflammatory tests altered, erythrocyte sedimentation rate: 45, C-reactive protein: 54,2. C3 and C4 normal. Cryoglobulins: nonreactive; protein electrophoresis: no monoclonal peaks; antinuclear antibodies: nonreactive; P-ANCA: 1:80 and C-ANCA: nonreactive; MPO-ANCA: positive; routine urine: macrohematuria, negative erythrocyte dysmorphism; $533.6 \mathrm{mg} /$ day proteinuria. He performed electroneurography that showed sensorimotor mononeuropathy multiplex, axonal, recent, with denervation, suggesting immunomediated cause. Biopsy of right sural nerve and right gastrocnemius muscle: small vessel vasculitis, nonnecrotizing arteritis in a small caliber artery, discrete inflammatory infiltrate with presence of eosinophils and neutrophils. Diagnosed with EGPA in January 2020. Pulse therapy was performed with methylprednisolone and cyclophosphamide with partial improvement of members pain, absence of constitutional symptoms and recovery of renal function. He kept outpatient follow-up and followed with monthly cyclophosphamide and corticoid gradual dose reduction, patient with progressive improvement, weight gain, paresthesia improvement.

\section{CONCLUSION}

Eosinophilic granulomatosis with polyangiitis is a disease that requires early suspicion, accurate diagnosis, aggressive treatment and periodic monitoring. It presents a diagnostic challenge since the syndrome is highly variable, but there are documented cases in which one of the main characteristics is absent. Eosinophilic granulomatosis with polyangiitis is rare in nonasthmatic individuals. Although in most of the series asthma is considered a mandatory criterion for the definition of the disease, today it is clear that its absence does not exclude the diagnosis. In addition, attention should be paid to patients who do not present previous atopic symptoms and who develop these symptoms together with eosinophilia, because when asthma appears very close to the vasculitic phase of the disease, the disease has often a worse prognosis. 